\title{
Lung Abscess Due to Candida Tropicalis in a HIV Positive Immunocompromised Patient: A Rare Case Report from Central India
}

\author{
Swati Manohar Bhise ${ }^{1}$, Arati Ankushrao Bhadade ${ }^{1 *}$, Bowalekar Sneha Sharad ${ }^{2}$ and Katkar Vyankatesh Jagannath ${ }^{1}$ \\ 'Department of Microbiology, Government Medical College, Nagpur(India) \\ ${ }^{2}$ Department of Microbiology, Lokmanya Tilak Medical college and Sion Hospital, Mumbai(India)
}

\section{ABSTRACT}

Invasive candidiasis is an emerging fungal infection in immunocompromised patients globally. Presentation of candidiasis in Human immunodeficiency virus (HIV) infected patients as lung abscess is very rare in tropical country like India. Here we report a case of lung abscess due to Candida tropicalis in a HIV positive immunocompromised patient. After diagnosis of C. tropicalis as pathogen the patient was treated by Amphotericin B and Fluconazole, the lung abscess got resolved but the patient succumbed to his illness may be due to nephrotoxicity of Amphotericin B and poor physical condition due other AIDS related comorbidities. The purpose of this article is to take note of this emerging fungal infection in India and also to review literature in this case.

Keywords: Lung Abscess, Non-albicans Candida, Candida Tropicalis, Human Immunodeficiency Virus, Immunocompromised Host

\section{Introduction}

Fungal infections remains a major cause of morbidity and mortality in HIV positive immunocompromised patients. ${ }^{[1]}$ In HIV infected patients most common manifestations of candida infections are oropharyngeal thrush, angular cheilitis, oesophagitis, balanitis but presentation as lung abscess is very rare. ${ }^{[1]}$ In over $90 \%$ cases of lung abscess polymicrobial bacteria can be found and the most common cause is anaerobic bacteria especially anaerobic streptococci. ${ }^{[2]}$ Although C. albicans has historically been most frequently isolated species, infections caused by the non-albicans candida (NAC) have been diagnosed with increasing frequency in recent years especially C. tropicalis. ${ }^{[3]}$ After doing extensive literature search previously only one case is reported of lung abscess due to C. tropicalis. ${ }^{[4]}$ Here we report a rare case of lung abscess due to $C$. tropicalis in a HIV positive immunocompromised patient.

\section{Case Report}

A 45 year old male patient, known case of benign prostatic hyperplasia was admitted to surgery unit with chief complaints of burning micturition and retention of urine since 15 days. The patient was also suffering from cough with expectoration since 3 weeks and low grade intermittent fever since 1 month. Expectoration was sticky white, non-foul smelling without hemoptysis. Patient was having history of chronic smoking, alcohol consumption and tobacco chewing since 20 years. Patient was HIV-1 positive since 15 years and having chronic oral candidiasis since 2 years. There was no history of other major illness in past.
On examination, patient was conscious and oriented. There was $5 \mathrm{~cm} \times 8 \mathrm{~cm}$ swelling in right supraclavicular region which was tender without any significant lymphadenopathy. On respiratory examination, patient was found to have shortness of breath increasing on exertion, not associated with tightness in chest, wheeze, paroxysmal nocturnal dyspnoea or orthopnoea. Bronchial breathing was positive on right side of chest.Total blood counts at admission were within normal range \& CD4 count was 350. Renal function tests done on admission were within normal range. Ultrasonography (USG) of prostate was suggestive of moderate prostatomegaly. USG of right supraclavicular region suggested inflammatory or infective etiology with small abscess. Radiograph of chest suggested consolidation of right middle and lower lateral lung lobes, right upper lobe cavity with air fluid level [Figure 1]. Fine Needle Aspiration Cytology of swelling suggested fungal etiology and fungal culture was advised.

In Sputum, bacteriological and mycobacterial culture revealed no growth. Aspirated lung abscess pus and Sputum examination for acid fast bacilli by Ziehl-Neelsen staining were negative. Gram staining of pus from right supraclavicular abscess as well as lung abscess showed presence of gram positive budding yeast cells. Pus culture and sputum fungal culture inoculated on Sabouraud dextrose Agar (SDA) incubated at $37^{\circ} \mathrm{C}$ grew creamy, white, pasty growth of Candida species within 2 days [Figure 2]. The Germ Tube test and Urease test were negative. On CHROM Agar for candida colonies were bluish pink colored colonies suggestive of $C$. tropicalis 
[Figure 3]. The Dalmau plate culture on cornmeal agar showed formation of blastoconidia in irregular clusters along with long pseudohyphae [Figure 4]. Patient was initially started on oral Nitrofurantoin which was later changed to injectable Ciprofloxacin and Metronidazole. On basis of clinical, radiological and microbiological evidences this patient was diagnosed as a case of lung abscess due to $C$. tropicalis. Hence, the patient was started on Amphotericin B $1 \mathrm{mg} / \mathrm{kg} /$ day intravenously and Tablet Fluconazole $150 \mathrm{mg}$ orally for 10 days. After 1 week renal function tests were deteriorated with serum urea $141 \mathrm{mg} / \mathrm{dl}$ and serum creatinine $1.5 \mathrm{mg} / \mathrm{dl}$. The patient continued on Tablet Fluconazole and showed significant improvement in lung abscess as well as right supraclavicular abscess evident by decrease in size of swelling for both, also relief of respiratory symptoms. However, general condition of the patient got deteriorated owing to raised renal function tests and he succumbed to his illness.

\section{Discussion}

In pre antibiotic era, lung abscess was caused by only one type of bacteria, and today almost all cases are caused by polymicrobial flora. ${ }^{[5]}$ Pulmonary candidiasis presenting as lung abscess is very rare. HIV is one of the major contributing factor for the increasing number of patients with fungal infections. ${ }^{[6]}$ Contributing factors for lung abscess are elderly, dental/periodontal infections (gingivitis-with bacterial concentration $>1011 / \mathrm{mL}$ ), alcoholism, drug abuse, diabetes mellitus, coma, artificial

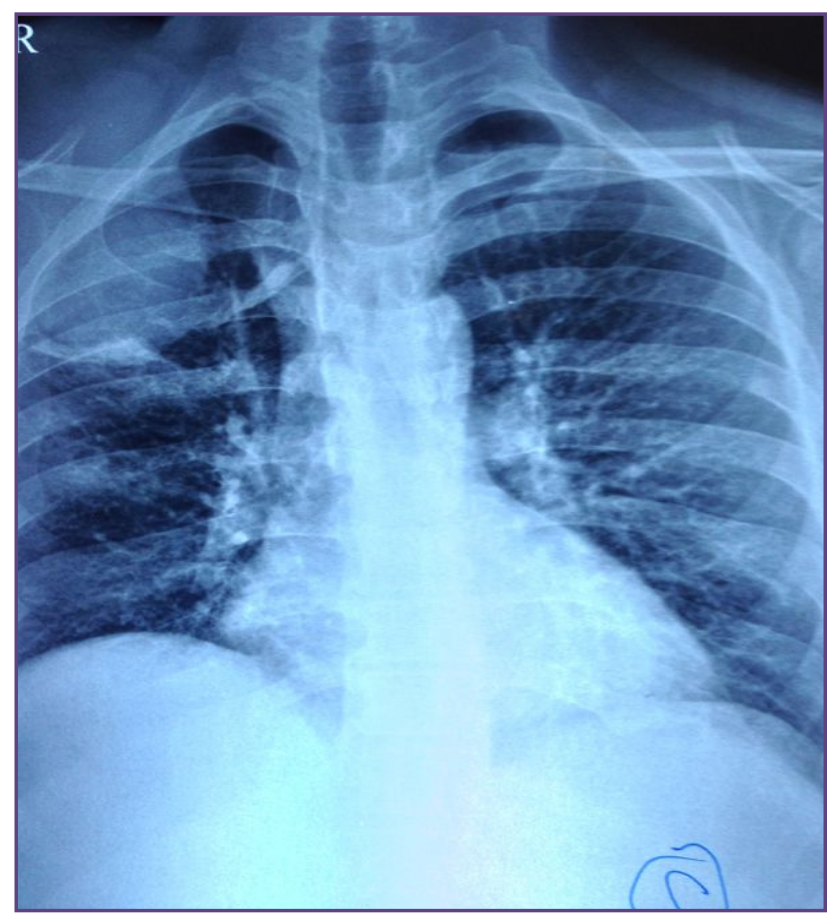

Fig. 1: X-ray chest PA view showing air fluid level in right upper lobe. ventilation, convulsions, neuromuscular disorders with bulbar dysfunctions, malnutrition, therapy with corticosteroids or immunosuppressants, mental retardation, gastro esophageal reflux disease, bronchial obstruction, inability to cough, sepsis. ${ }^{[7]}$

In our case, the patient was immunocompromised due to HIV infection and prone for opportunistic infection due to low CD4 count. Disseminated C. tropicalis infection has been reported in immunocompromised patients who have chronic mucocutaneous candidiasis. ${ }^{[8]}$ In our case chronic oral candidiasis was present in patient hence this might be the source of infection. A particular problem with fungal lung abscess patients is that they are difficult to distinguish clinically from patients with bacterial sepsis which leads to delay in diagnosis and delay in antifungal therapy. ${ }^{[4]}$ Once candida infection is confirmed, species level identification is must for prediction of antifungal susceptibility. ${ }^{[9]} C$. tropicalis have moderate level fluconazole resistant hence other antifungal drugs like Amphotericin B or Voriconazole should be considered for treatment. Amphotericin B have very high nephrotoxicity hence renal function tests should be monitored while treatment to reduce the mortality. ${ }^{[10]}$ To our knowledge there are very few cases of lung abscesses due to candida infection. ${ }^{[4,11,12]}$ Gupta A et al (2013) reported a case of 45 year immunosuppressed male patient with history of renal transplant 5 years back. Patient was diagnosed as having $C$. tropicalis lung abscess and started on Amphotericin B but the patient died due to complications.

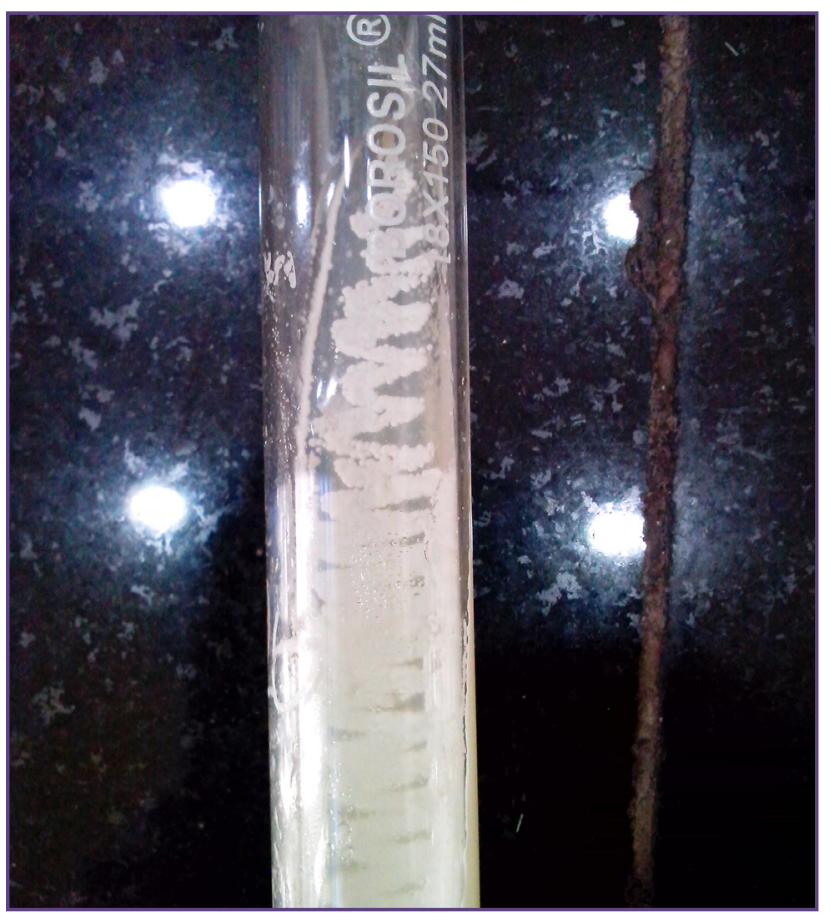

Fig. 2: SDA showing yeasty pasty colony of Candida tropicalis. 


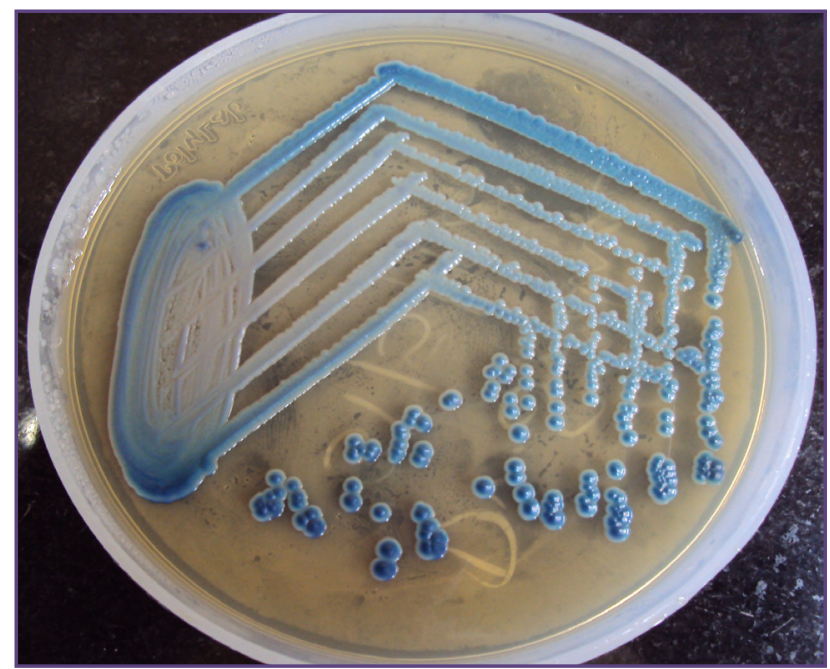

Fig. 3: CHROME agar showing bluish pink colonies of $\mathrm{C}$. tropicalis.

${ }^{[4]}$ Rubin AE et al (1977) reported a case of lung abscess due to $C$. albicans in 49 year old immunocompromised patient having history of spleenectomy, Hodgkin's disease and was receiving immunosuppressive therapy. After treatment with 5-Fluorocytosine lung abscess resolved and patient survived. ${ }^{[1]}$ Gupta $P$ et al (2016) reported a case of 42 year male patient of lung abscess due to $C$. albicans but it was present in immunocompetent patient and after antifungal treatment patient improved clinically and radiologically. ${ }^{[12]}$

\section{Conclusion}

Lung abscess due to $C$. tropicalis in HIV infected immunocompromised patient is very rare. A high index of suspicion for a fungal cause due to non-albicans candida species in such immunocompromised individuals is required. Adequate antifungal therapy with correct dosage and duration should be instituted early in such cases. By keeping in mind renal toxicity of Amphotericin B, its dosage should be carefully adjusted by regular renal function tests monitoring.

\section{Reference}

1. Chander J. Textbook of Medical Mycology.13th ed. New Delhi: Mehta publishers.2012 p.522-530.

2. Takayanagi N, Kagiyama N, Ishiguro T, Tokunaga D, Sugita Y. Etiology and outcome of community-acquired lung abscess. Respiration2010; 80(2):98-105.

3. Goel N, Ranjan P, Aggarwal R, ChaudharyJ, Nanda S.

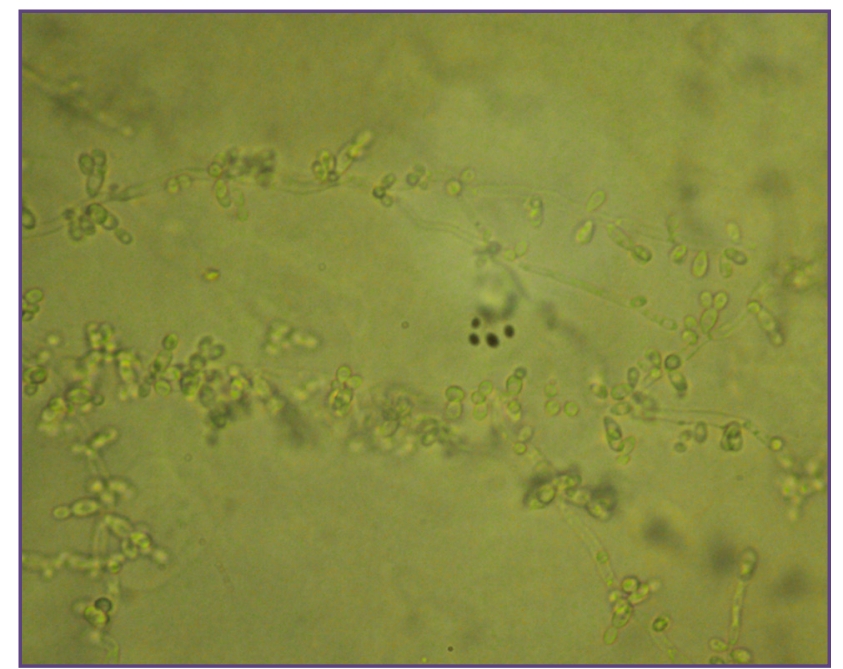

Fig. 4: Cornmeal agar showing irregular clusters of blastoconidia with long pseudohyphae (400x magnification). Emergence of Non-albicans candida in neonatal septicaemia and antifugal susceptibility: Experience from a tertiary care center. Journal of Laboratory Physicians. 2009; 1(2):53-55.

4. Gupta A, Bhowmik D, Dogra P, Mendonca S, Gupta A. Candia lung abscess in renal transplant recipient. Saudi J Kidney Dis Transpl .2013;24(2): 315-7.

5. Yazbeck MF, Dahdel M, Kalra A, et al. Lung abscess: update on microbiology and management. Am J Ther 2014;21:217-21.

6. Nissapatorn V, Lee C, Fatt QK, Abdullah KA. AIDS-related opportunistic infections in Hospital Kuala Lumpur. Jpn J Infect Dis. 2003; 56: 187-92.

7. Ando K, Okhuni Y, Matsunuma R, et al. Prognostic lung abscess factors. Kansenshogaku Zasshi 2010;84:425-30.

8. Dixon TC, Steinbach WJ, Benjamin DK, Williams LW, Myers LA.. Disseminated Candida tropicalis in a patient with chronic mucocutaneous candidiasis. South Med J. 2004;97: 788-790.

9. Fleck R, Dietz A, Hof H. In vitro susceptibility of Candida species to five antifungal agents in a German university hospital assessed by the reference broth microdilution method and Etest. J Antimicrob Chemother 2007; 59: 767-71.

10. Gilbert D. Amphotericin B nephrotoxicity. Journal of Antimicrobial chemotherapy. 2002; 49:37-41.

11. Rubin AE and Alroy GG. Candida albicans abscess of lung. Thorax. 1977 1977; 32:373-376.

12. Gupta P, Agarwal D, Yadav R. lung abscess due to pulmonary candidiasis. Lung India .2006; 23: 160-162.

*Corresponding author:

Dr. Arati Ankuhrao Bhadade, 303, Dwarkamai apartment, near Dargah, Wanjari Nagar, Nagpur,-440003(India),

Phone: +91 9975933877

Email: aaratimbbs@gmail.com

Financial or other Competing Interests: None. 